Bull. Korean Math. Soc. 51 (2014), No. 3, pp. 847-862

http://dx.doi.org/10.4134/BKMS.2014.51.3.847

\title{
FINITE ELEMENT APPROXIMATIONS OF \\ THE OPTIMAL CONTROL PROBLEMS FOR STOCHASTIC STOKES EQUATIONS
}

\author{
Youngmi Choi, Soohyun Kim, and Hyung-Chun Lee
}

\begin{abstract}
Finite element approximation solutions of the optimal control problems for stochastic Stokes equations with the forcing term perturbed by white noise are considered. Error estimates are established for the fully coupled optimality system using Brezzi-Rappaz-Raviart theory Numerical examples are also presented to examine our theoretical results.
\end{abstract}

\section{Introduction}

The optimal control problems for partial differential equations have been studied widely (see $[1,11,12,15,16,18,20,21,22,23]$ ). Recently there has been an increased interest in mathematical analyses and computations of stochastic partial differential equations (see $[3,4,5,13,19,25,26,27])$. In the article [7], numerical solutions of the stochastic Stokes equations driven by white noise perturbed forcing terms using finite element methods was considered. We use their results to analyze our optimal control problems for stochastic Stokes equations.

The optimal control problem we consider here is to minimize the functional

$$
\mathcal{J}(\mathbf{u}, p, \mathbf{f})=\mathbb{E}\left(\frac{1}{2} \int\left|\mathbf{u}-\mathbf{U}_{d}\right|^{2} d x+\frac{\delta}{2} \int|\mathbf{f}|^{2} d x\right)
$$

subject to the steady-state Stokes equations with the forcing term perturbed by white noise:

$$
\begin{aligned}
-\nu \Delta \mathbf{u}+\nabla p & =\mathbf{f}+\sigma \dot{W} \quad \text { in } \Omega, \\
\nabla \cdot \mathbf{u} & =0 \quad \text { in } \Omega \\
\mathbf{u} & =\mathbf{0} \quad \text { on } \partial \Omega
\end{aligned}
$$

Received May 12, 2013; Revised August 31, 2013.

2010 Mathematics Subject Classification. 49A22, 49B22, 65N30.

Key words and phrases. stochastic Stokes equations, optimal control, white noise.

This work was supported by Korea Research Foundation under the grant number NRF2010-0026032 and NRF-2010-0010013. 
where $\mathbf{U}_{d}$ is a given desired function. Here, $\Omega$ is a convex polygon in $\mathbb{R}^{2}$ or a convex polyhedron in $\mathbb{R}^{3}, \mathbf{u}$ is a velocity of the fluid flow, $p$ is the pressure, $\mathbf{f}$ is a prescribed forcing term, $\nu$ is the viscous constant, $\sigma$ is a positive continuous function in $\Omega$ and $\dot{W}=\left(\dot{W}^{1}, \ldots, \dot{W}^{d}\right)$ is the white noise such that

$$
\mathbb{E}\left(\dot{W}^{j}(x) \dot{W}^{j}\left(x^{\prime}\right)\right)=\delta\left(x-x^{\prime}\right), \quad x, x^{\prime} \in \Omega, \quad j=1, \ldots, d,
$$

where $\delta$ denotes the usual Dirac delta function and $\mathbb{E}$ the expectation. We assume that $\dot{W}^{i}$ and $\dot{W}^{j}, i \neq j, i, j=1, \ldots, d$, are independent. We also assume that $p$ satisfies the zero mean constraint, $\int_{\Omega} p d x=0$. The objective of this optimal control problem is to seek a state variables $\mathbf{u}$ and $p$, and the control $\mathbf{f}$ which minimize the expectation of $L^{2}$-norm distances between $\mathbf{u}$ and $\mathbf{U}_{d}$ and satisfy (2). The second term in (1) is added as a limiting the cost of control and the positive penalty parameter $\delta$ can be used to change the relative importance of the two terms appearing in the definition of the functional.

The plan of the paper is as follows. In the next section, we define an approximate solution of the optimal control problems for the stochastic Stokes equations by discretizing the white noise and show there is an optimal solution for the optimization problem. Then we derive the optimality system of equations by the Gâteaux differentiability. In Section 3, we construct finite element approximations for the stochastic Stokes equations and carry out the error analysis using Brezzi-Rappaz-Raviart theory. Finally, in Section 4, we present numerical simulation results using the algorithm constructed in Section 3.

\subsection{Notations}

We use standard Sobolev space notation (see [2]). The standard Sobolev spaces $H^{m}(\Omega)$ and $H_{0}^{1}(\Omega)$ will be used with the associated standard inner products $(\cdot, \cdot)_{m}$ and their respective norms $\|\cdot\|_{m}$ where

$$
H_{0}^{1}(\Omega)=\left\{f \in H^{1}(\Omega) \mid f=0 \text { on } \partial \Omega\right\} .
$$

In particular, for $m=0$ we replace $H^{m}(\Omega)$ and $H_{0}^{1}(\Omega)$ by $L^{2}(\Omega)$ and $L_{0}^{2}(\Omega)$ with the norm $\|\cdot\|$ and inner product $(\cdot, \cdot)$ where

$$
L_{0}^{2}(\Omega)=\left\{f \in L^{2}(\Omega) \mid \int_{\Omega} f(x) d \Omega=0\right\} .
$$

For positive values of $m$ the space $H^{-m}(\Omega)$ is defined as the dual space of $H_{0}^{m}(\Omega)$ equipped with the norm $\|\phi\|_{-m}=\sup _{0 \neq v \in H_{0}^{m}(\Omega)} \frac{\langle\phi, v\rangle}{\|v\|_{m}}$ where $\langle\cdot, \cdot\rangle$ is the duality pairing between $H^{-m}(\Omega)$ and $H_{0}^{m}(\Omega)$. For vector valued functions, we define the Sobolev space $\mathbf{H}^{m}(\Omega)=\left[H^{m}(\Omega)\right]^{k}, k=2$ or 3 .

\subsection{Approximation with discretized white noise}

In this subsection we define an approximate solution of (2) by discretizing the white noise $\dot{W}$. First we introduce a discretization for the white noise. Let $\left\{T_{h}\right\}$ be a family of triangulations of $\bar{\Omega}$, where $h \in(0,1)$ is the meshsize. We 
assume that the family is quasiuniform, i.e., there exist positive constants $\rho_{1}$ and $\rho_{2}$ such that

$$
\rho_{1} h \leq R_{T}^{\mathrm{inr}}<R_{T}^{\mathrm{cir}} \leq \rho_{2} h, \quad \forall T \in T_{h}, \quad \forall 0<h<1,
$$

where $R_{T}^{\mathrm{inr}}$ and $R_{T}^{\mathrm{cir}}$ are the inradius and the circumradius of $T$. Write

$$
\xi_{T}^{j}=\frac{1}{\sqrt{|T|}} \int_{T} 1 d W^{j}(x), \quad j=1, \ldots, d
$$

for each triangle $T \in T_{h}$, where $|T|$ denotes the area of $T$. Then the piecewise constant approximation to $\dot{W}^{j}(x)$ is given by

$$
\dot{W}_{h}^{j}(x)=\sum_{T \in T_{h}}|T|^{-\frac{1}{2}} \xi_{T}^{j} \chi_{T}(x),
$$

where $\chi_{T}$ is the characteristic function of $T$. It is apparent that $\dot{W}_{h}=\left(\dot{W}_{h}^{1}, \ldots\right.$, $\left.\dot{W}_{h}^{d}\right) \in\left(L^{2}(\Omega)\right)^{d}$ almost surely. However, we have the following estimate, in [8], which shows that $\left\|\dot{W}_{h}\right\|$ is unbounded as $h \rightarrow 0$.

Lemma 1.1. Let $\left\|\dot{W}_{h}\right\|=\sqrt{\left\|\dot{W}_{h}^{1}\right\|^{2}+\cdots+\left\|\dot{W}_{h}^{d}\right\|^{2}}$. Then there exist positive constant $C_{1}$ and $C_{2}$ independent of $h$ such that

$$
C_{1} h^{-k} \leq \mathbb{E}\left(\left\|\dot{W}_{h}\right\|^{2}\right) \leq C_{2} h^{-k}
$$

where $k=2$ or 3 for $d=2$ or 3 , respectively.

Now we consider the approximation problem for (2) with the discretized white noise forcing term $\dot{W}_{h}$ :

$$
\begin{aligned}
-\nu \Delta \mathbf{u}_{h}+\nabla p_{h} & =\mathbf{f}_{h}+\sigma \dot{W}_{h} \quad \text { in } \Omega, \\
\nabla \cdot \mathbf{u}_{h} & =0 \quad \text { in } \Omega, \\
\mathbf{u}_{h} & =\mathbf{0} \quad \text { on } \partial \Omega .
\end{aligned}
$$

We have the following estimate concerning the bounds for $\mathbf{u}_{h}$ and $p_{h}$.

Lemma 1.2. There exists a positive constant $C$ independent of $h$ such that

$$
\mathbb{E}\left(\left\|\mathbf{u}_{h}\right\|_{2}^{2}+\left\|p_{h}\right\|_{1}^{2}\right) \leq C\left(\left\|\mathbf{f}_{h}\right\|^{2}+h^{-k}\right),
$$

where $k=2$ or 3 for $d=2$ or 3 , respectively.

Proof. From the standard estimates of Stokes equations (see e.g., [14]) and Lemma 1.1, we have that

$$
\mathbb{E}\left(\left\|\mathbf{u}_{h}\right\|_{2}^{2}+\left\|p_{h}\right\|_{1}^{2}\right) \leq C \mathbb{E}\left(\left\|\mathbf{f}_{h}\right\|^{2}+\left\|\dot{W}_{h}\right\|^{2}\right) \leq C\left(\left\|\mathbf{f}_{h}\right\|^{2}+h^{-k}\right),
$$

where $k=2$ or 3 for $d=2$ or 3 , respectively.

For the errors $\mathbf{u}-\mathbf{u}_{h}$ and $p-p_{h}$, we have the following estimate which is in [7]. 
Theorem 1.3. Let $(\mathbf{u}, p)$ and $\left(\mathbf{u}_{h}, p_{h}\right)$ be the solution of (2) and (3), respectively. Then there exists a constant $C$ such that

$$
\mathbb{E}\left(\left\|\mathbf{u}-\mathbf{u}_{h}\right\|^{2}+\left\|p-p_{h}\right\|_{-1}^{2}\right) \leq C|\ln h| h^{2}
$$

for $d=2$ and

$$
\mathbb{E}\left(\left\|\mathbf{u}-\mathbf{u}_{h}\right\|^{2}+\left\|p-p_{h}\right\|_{-1}^{2}\right) \leq C h
$$

for $d=3$

\section{The optimal control problem}

\subsection{The optimization problem}

Let $\mathbf{u}_{h} \in \mathbf{H}_{0}^{1}(\Omega)$ and $p_{h} \in L_{0}^{2}(\Omega)$ denote the state variables, and let $\mathbf{f}_{h} \in$ $\mathbf{H}^{-1}(\Omega)$ denote the distributed control. The state and control variables are also constrained to satisfy the system (3), which recast into the weak form:

$$
\begin{aligned}
\nu a\left(\mathbf{u}_{h}, \mathbf{v}\right)+b\left(\mathbf{v}, p_{h}\right) & =\left\langle\mathbf{f}_{h}, \mathbf{v}\right\rangle+\left(\sigma \dot{W}_{h}, \mathbf{v}\right) \quad \forall \mathbf{v} \in \mathbf{H}_{0}^{1}(\Omega), \\
b\left(\mathbf{u}_{h}, q\right) & =0 \quad \forall q \in L_{0}^{2}(\Omega),
\end{aligned}
$$

where

$$
\begin{aligned}
a(\mathbf{u}, \mathbf{v})=\int_{\Omega} \nabla \mathbf{u}: \nabla \mathbf{v} d \Omega & \forall \mathbf{u}, \mathbf{v} \in \mathbf{H}^{1}(\Omega), \\
b(\mathbf{v}, q)=-\int_{\Omega} \nabla \cdot \mathbf{v} q d \Omega & \forall \mathbf{v} \in \mathbf{H}^{1}(\Omega), \forall q \in L^{2}(\Omega) .
\end{aligned}
$$

With $\mathcal{J}\left(\mathbf{u}_{h}, p_{h}, \mathbf{f}_{h}\right)$ given by (1), the admissibility set $\mathcal{U}_{a d}$ is defined by

$$
\begin{aligned}
\mathcal{U}_{a d}=\left\{\left(\mathbf{u}_{h}, p_{h}, \mathbf{f}_{h}\right)\right. & \in \mathbf{H}_{0}^{1}(\Omega) \times L_{0}^{2}(\Omega) \times \mathbf{H}^{-1}(\Omega): \\
& \left.\mathcal{J}\left(\mathbf{u}_{h}, p_{h}, \mathbf{f}_{h}\right)<\infty \text { and }\left(\mathbf{u}_{h}, p_{h}, \mathbf{f}_{h}\right) \text { satisfies (4) and (5) }\right\} .
\end{aligned}
$$

Then $\left(\hat{\mathbf{u}}_{h}, \hat{p}_{h}, \hat{\mathbf{f}}_{h}\right) \in \mathcal{U}_{a d}$ is called an optimal solution if there exists $\epsilon>0$ such that

$$
\mathcal{J}\left(\hat{\mathbf{u}}_{h}, \hat{p}_{h}, \hat{\mathbf{f}}_{h}\right) \leq \mathcal{J}\left(\mathbf{u}_{h}, p_{h}, \mathbf{f}_{h}\right) \quad \forall\left(\mathbf{u}_{h}, p_{h}, \mathbf{f}_{h}\right) \in \mathcal{U}_{a d}
$$

satisfying

$$
\left\|\hat{\mathbf{u}}_{h}-\mathbf{u}_{h}\right\|_{1}+\left\|\hat{p}_{h}-p_{h}\right\|+\left\|\hat{\mathbf{f}}_{h}-\mathbf{f}_{h}\right\|_{-1}<\epsilon .
$$

The optimal control problem can now be formulated as a constrained minimization problem in a Hilbert space

$$
\min _{\left(\mathbf{u}_{h}, p_{h}, \mathbf{f}_{h}\right) \in \mathcal{U}_{a d}} \mathcal{J}\left(\mathbf{u}_{h}, p_{h}, \mathbf{f}_{h}\right) .
$$

The existence and uniqueness of an optimal solution of (6) is easily proven using standard arguments in the following theorem (see [20]).

Theorem 2.1. There is an optimal solution $\left(\hat{\mathbf{u}}_{h}, \hat{p}_{h}, \hat{\mathbf{f}_{h}}\right) \in \mathcal{U}_{\text {ad }}$ of $\mathcal{J}\left(\mathbf{u}_{h}, p_{h}, \mathbf{f}_{h}\right)$. 
Proof. We first note that $\mathcal{U}_{a d}$ is clearly not empty. Let $\left\{\left(\mathbf{u}_{h}^{(n)}, p_{h}^{(n)}, \mathbf{f}_{h}^{(n)}\right)\right\}$ be a minimizing sequence in $\mathcal{U}_{a d}$, that is,

$$
\lim _{n \rightarrow \infty} \mathcal{J}\left(\mathbf{u}_{h}^{(n)}, p_{h}^{(n)}, \mathbf{f}_{h}^{(n)}\right)=\inf _{\left(\mathbf{u}_{h}, p_{h}, \mathbf{f}_{h}\right) \in \mathcal{U}_{a d}} \mathcal{J}\left(\mathbf{u}_{h}, p_{h}, \mathbf{f}_{h}\right) .
$$

Because a convergent sequence is bounded, we have that $\left\|\mathbf{f}_{h}^{(n)}\right\|_{-1} \leq C$ for some $C>0$. That is, the sequence $\left\{\mathbf{f}_{h}^{(n)}\right\}$ is uniformly bounded in $\mathbf{H}^{-1}(\Omega)$. Thus there is a subsequence $\left\{\mathbf{f}_{h}^{\left(n_{i}\right)}\right\}$ of $\left\{\mathbf{f}_{h}^{(n)}\right\}$ and $\hat{\mathbf{f}}_{h} \in \mathbf{H}^{-1}(\Omega)$ such that for any $\mathbf{v}_{h} \in \mathbf{H}_{0}^{1}(\Omega)$,

$$
\left\langle\mathbf{f}_{h}^{\left(n_{i}\right)}, \mathbf{v}_{h}\right\rangle \rightarrow\left\langle\hat{\mathbf{f}}_{h}, \mathbf{v}_{h}\right\rangle .
$$

Also, using the bound $\left\|\mathbf{u}_{h}\right\|_{1}+\left\|p_{h}\right\| \leq C\left(\left\|\mathbf{f}_{h}\right\|_{-1}+\left\|\dot{W}_{h}\right\|\right)$, we have that the sequence $\left\{\left\|\mathbf{u}_{h}^{(n)}\right\|_{1}\right\}$ and $\left\{\left\|p_{h}^{(n)}\right\|\right\}$ is uniformly bounded. So, we may then extract subsequences such that

$$
\begin{array}{rll}
p_{h}^{\left(n_{i}\right)} \rightarrow \hat{p}_{h} & \text { in } & L_{0}^{2}(\Omega), \\
\mathbf{u}_{h}^{\left(n_{i}\right)} \rightarrow \hat{\mathbf{u}}_{h} & \text { in } & \mathbf{H}_{0}^{1}(\Omega), \\
\mathbf{u}_{h}^{\left(n_{i}\right)} \rightarrow \hat{\mathbf{u}}_{h} & \text { in } & \mathbf{L}_{0}^{2}(\Omega),
\end{array}
$$

for some $\left(\hat{\mathbf{u}}_{h}, \hat{p}_{h}, \hat{\mathbf{f}}_{h}\right) \in \mathbf{H}_{0}^{1}(\Omega) \times L_{0}^{2}(\Omega) \times \mathbf{H}^{-1}(\Omega)$. The last convergence results above follows from the compact imbedding $\mathbf{H}_{0}^{1}(\Omega) \hookrightarrow \hookrightarrow \mathbf{L}_{0}^{2}(\Omega)$. We may then easily pass to the limit in $(4)-(5)$. Now, by the weak lower semi-continuity of $\mathcal{J}(\cdot, \cdot, \cdot)$, we conclude that $\left(\hat{\mathbf{u}}_{h}, \hat{p}_{h}, \hat{\mathbf{f}}_{h}\right)$ is an optimal solution, i.e.,

$$
\inf _{\left(\mathbf{u}_{h}, p_{h}, \mathbf{f}_{h}\right) \in \mathcal{U}_{a d}} \mathcal{J}\left(\mathbf{u}_{h}, p_{h}, \mathbf{f}_{h}\right)=\lim _{i \rightarrow \infty} \inf \mathcal{J}\left(\mathbf{u}^{\left(n_{i}\right)}, p^{\left(n_{i}\right)}, \mathbf{f}^{\left(n_{i}\right)}\right)=\mathcal{J}\left(\hat{\mathbf{u}}_{h}, \hat{p}_{h}, \hat{\mathbf{f}}_{h}\right) .
$$

Thus, we have shown that an optimal solution belonging to $\mathcal{U}_{a d}$ exists. Finally, the uniqueness of the optimal solution follows from the convexity of the functional and the linearity of the constraint equations.

\subsection{The optimality system}

Assume that $\hat{\mathbf{f}}_{h} \in \mathbf{L}_{0}^{2}(\Omega)$ is a minimizer of $\mathcal{J}$ and $\hat{\mathbf{u}}_{h}$ and $\hat{p}_{h}$ are the corresponding state variables. Define the adjoint variables $\hat{\mathbf{v}}_{h}$ and $\hat{q}_{h}$ such that

$$
\begin{aligned}
\nu a\left(\hat{\mathbf{v}}_{h}, \mathbf{z}\right)+b\left(\mathbf{z}, \hat{q}_{h}\right) & =\left(\hat{\mathbf{u}}_{h}-\mathbf{U}_{d}, \mathbf{z}\right) \quad \forall \mathbf{z} \in \mathbf{H}_{0}^{1}(\Omega), \\
b\left(\hat{\mathbf{v}}_{h}, s\right) & =0 \quad \forall s \in L_{0}^{2}(\Omega) .
\end{aligned}
$$

Theorem 2.2. $\mathcal{J}$ has a unique minimizer $\hat{\mathbf{f}}_{h} \in \mathbf{L}_{0}^{2}(\Omega)$ and it is determined by

$$
\delta \int_{\Omega} \hat{\mathbf{f}}_{h} \mathbf{g} d x=-\int_{\Omega} \hat{\mathbf{v}}_{h} \mathbf{g} d x \quad \forall \mathbf{g} \in \mathbf{L}_{0}^{2}(\Omega) .
$$

Proof. The existence and uniqueness follow from the standard theory of optimal controls (see [24]). 
For $\mathbf{f}_{h} \in \mathbf{L}_{0}^{2}(\Omega)$, let $\left(\mathbf{u}_{h}\left(\mathbf{f}_{h}\right), p_{h}\left(\mathbf{f}_{h}\right)\right)$ be the solution of (4)-(5). Then $\forall \mathbf{g} \in$ $\mathbf{L}^{2}(\Omega)$,

$$
\left.\frac{\partial}{\partial \epsilon} \mathbf{u}_{h}\left(\hat{\mathbf{f}}_{h}+\epsilon \mathbf{g}\right)\right|_{\epsilon=0}=\mathbf{u}_{h}(\mathbf{g}) .
$$

Since $\hat{\mathbf{f}}_{h}$ is a minimizer of $\mathcal{J}$,

$$
\frac{d \mathcal{J}\left(\hat{\mathbf{u}}_{h}\left(\hat{\mathbf{f}}_{h}\right), \hat{p}_{h}\left(\hat{\mathbf{f}}_{h}\right), \hat{\mathbf{f}}_{h}\right)}{d \mathbf{f}_{h}}=\left.\frac{d}{d \epsilon} \mathcal{J}\left(\hat{\mathbf{u}}_{h}\left(\hat{\mathbf{f}}_{h}+\epsilon \mathbf{g}\right), \hat{p}_{h}\left(\hat{\mathbf{f}}_{h}+\epsilon \mathbf{g}\right), \hat{\mathbf{f}}_{h}+\epsilon \mathbf{g}\right)\right|_{\epsilon=0}=0
$$

which implies that

$$
\int_{\Omega}\left(\hat{\mathbf{u}}_{h}-\mathbf{U}_{d}\right) \hat{\mathbf{u}}_{h}(\mathbf{g}) d x+\delta \int_{\Omega} \hat{\mathbf{f}}_{h} \mathbf{g} d x=0 .
$$

By (7) and integration by parts, we have that

$$
\begin{aligned}
\int_{\Omega}\left(\hat{\mathbf{u}}_{h}-\mathbf{U}_{d}\right) \hat{\mathbf{u}}_{h}(\mathbf{g}) d x & =\nu \int_{\Omega} \nabla \hat{\mathbf{v}}_{h} \nabla \hat{\mathbf{u}}_{h}(\mathbf{g}) d x+\int_{\Omega} \nabla \cdot \hat{\mathbf{u}}_{h}(\mathbf{g}) \hat{q}_{h} d x \\
& =-\int_{\Omega} \hat{\mathbf{v}}_{h} \nabla \cdot\left(\nu \nabla \hat{\mathbf{u}}_{h}(\mathbf{g})\right) d x \\
& =\int_{\Omega} \hat{\mathbf{v}}_{h} \mathbf{g} d x,
\end{aligned}
$$

almost surely. Since $\mathbf{g} \in \mathbf{L}_{0}^{2}(\Omega)$ is arbitrary, we obtain (8).

From the above theorem, we conclude that solving the minimization problems (6) is equivalent to solving the following optimality system of equations.

$$
\left\{\begin{aligned}
\nu a\left(\mathbf{u}_{h}, \mathbf{w}\right)+b\left(\mathbf{w}, p_{h}\right)=\left\langle\mathbf{f}_{h}, \mathbf{w}\right\rangle & +\left(\sigma \dot{W}_{h}, \mathbf{w}\right) \quad \forall \mathbf{w} \in \mathbf{H}_{0}^{1}(\Omega), \\
b\left(\mathbf{u}_{h}, r\right) & =0 \quad \forall r \in L_{0}^{2}(\Omega), \\
\nu a\left(\mathbf{v}_{h}, \mathbf{z}\right)+b\left(\mathbf{z}, q_{h}\right) & =\left(\mathbf{u}_{h}-\mathbf{U}_{d}, \mathbf{z}\right) \quad \forall \mathbf{z} \in \mathbf{H}_{0}^{1}(\Omega), \\
b\left(\mathbf{v}_{h}, s\right) & =0 \quad \forall s \in L_{0}^{2}(\Omega) \\
\delta\left(\mathbf{f}_{h}, \mathbf{g}\right)+\left(\mathbf{v}_{h}, \mathbf{g}\right) & =0 \quad \forall \mathbf{g} \in \mathbf{L}_{0}^{2}(\Omega) .
\end{aligned}\right.
$$

Using the optimality condition $\mathbf{f}_{h}=-\frac{\mathbf{v}_{h}}{\delta}$, we obtain the following optimality system: find $\left(\mathbf{u}_{h}, p_{h}, \mathbf{v}_{h}, q_{h}\right) \in \mathbf{H}_{0}^{1}(\Omega) \times L_{0}^{2}(\Omega) \times \mathbf{H}_{0}^{1}(\Omega) \times L_{0}^{2}(\Omega)$ such that

$$
\left\{\begin{aligned}
\nu a\left(\mathbf{u}_{h}, \mathbf{w}\right)+b\left(\mathbf{w}, p_{h}\right)=\left(-\frac{\mathbf{v}_{h}}{\delta}, \mathbf{w}\right) & +\left(\sigma \dot{W}_{h}, \mathbf{w}\right) \quad \forall \mathbf{w} \in \mathbf{H}_{0}^{1}(\Omega), \\
b\left(\mathbf{u}_{h}, r\right) & =0 \quad \forall r \in L_{0}^{2}(\Omega), \\
\nu a\left(\mathbf{v}_{h}, \mathbf{z}\right)+b\left(\mathbf{z}, q_{h}\right) & =\left(\mathbf{u}_{h}-\mathbf{U}_{d}, \mathbf{z}\right) \quad \forall \mathbf{z} \in \mathbf{H}_{0}^{1}(\Omega), \\
b\left(\mathbf{v}_{h}, s\right) & =0 \quad \forall s \in L_{0}^{2}(\Omega) .
\end{aligned}\right.
$$




\section{Finite element approximations}

\subsection{Finite element discretizations}

We consider the numerical approximations of (9) using the finite element method. First we choose a family of finite dimensional subspaces $\mathbf{V}_{h} \subset \mathbf{H}^{1}(\Omega)$ and $Q_{h} \subset L^{2}(\Omega)$. We let $\mathbf{V}_{h}^{0}=\mathbf{V}_{h} \cap \mathbf{H}_{0}^{1}(\Omega)$ and $Q_{h}^{0}=Q_{h} \cap L_{0}^{2}(\Omega)$. These families are parameterized by a parameter $h$ that tends to zero; commonly, $h$ is chosen to be some measure of the grid size. First, we have the approximation properties: there exist a constant $C$, independent of $h, \mathbf{v}_{h}$, and $q_{h}$, such that

$$
\begin{aligned}
& \inf _{\hat{\mathbf{v}}_{h} \in \mathbf{V}_{h}}\left\|\mathbf{v}-\hat{\mathbf{v}}_{h}\right\|_{s} \leq C h^{2-s}\|\mathbf{v}\|_{2} \quad s=0,1, \forall \mathbf{v} \in \mathbf{H}^{2}(\Omega), \\
& \inf _{\hat{q}_{h} \in Q_{h}}\left\|q-\hat{q}_{h}\right\| \leq C h\|q\|_{1} \quad \forall q \in H^{1}(\Omega) .
\end{aligned}
$$

Next, we assume the inf-sup condition: there exists a constant $C$, independent of $h$, such that

$$
\inf _{0 \neq \hat{q}_{h} \in Q_{h}} \sup _{0 \neq \hat{\mathbf{v}}_{h} \in \mathbf{V}_{h}} \frac{b\left(\hat{\mathbf{v}}_{h}, \hat{q}_{h}\right)}{\left\|\hat{\mathbf{v}}_{h}\right\|_{1}\left\|\hat{q}_{h}\right\|_{0}} \geq C .
$$

The finite element approximation for (9) is to find $\hat{\mathbf{u}}_{h}, \hat{p}_{h}, \hat{\mathbf{v}}_{h}$ and $\hat{q}_{h}$ such that

$$
\left\{\begin{aligned}
\nu a\left(\hat{\mathbf{u}}_{h}, \mathbf{w}_{h}\right)+b\left(\mathbf{w}_{h}, \hat{p}_{h}\right) & =\left(-\frac{\hat{\mathbf{v}}_{h}}{\delta}, \mathbf{w}_{h}\right)+\left(\sigma \dot{W}_{h}, \mathbf{w}_{h}\right) \quad \forall \mathbf{w}_{h} \in \mathbf{V}_{h}^{0}, \\
b\left(\hat{\mathbf{u}}_{h}, r_{h}\right) & =0 \quad \forall r_{h} \in Q_{h}^{0}, \\
\nu a\left(\hat{\mathbf{v}}_{h}, \mathbf{z}_{h}\right)+b\left(\mathbf{z}_{h}, \hat{q}_{h}\right) & =\left(\hat{\mathbf{u}}_{h}-\mathbf{U}_{d}, \mathbf{z}_{h}\right) \quad \forall \mathbf{z}_{h} \in \mathbf{V}_{h}^{0}, \\
b\left(\hat{\mathbf{v}}_{h}, s_{h}\right) & =0 \quad \forall s_{h} \in Q_{h}^{0} .
\end{aligned}\right.
$$

\subsection{Discretization error estimates}

The B-R-R theory in [6] implies that the error of approximation of solutions of certain nonlinear problems under certain hypotheses is basically the same as the error of approximation of solutions of related linear problems (see [6], [14], [19]). We first fit our optimality system and its discrete approximation into the B-R-R framework. Then we obtain the desired error estimates on the solution of the optimality system of equations by verifying each assumption of the B-R-R theory. For this purpose, we show how to cast nonlinear problems (9) and (10) in the respective canonical forms

$$
F\left(\lambda, \psi_{h}\right) \equiv \psi_{h}+T G\left(\lambda, \psi_{h}\right)=0,
$$

and

$$
F_{h}\left(\lambda, \hat{\psi}_{h}\right) \equiv \hat{\psi}_{h}+T_{h} G\left(\lambda, \hat{\psi}_{h}\right)=0 .
$$

Let $\lambda=\frac{1}{\nu}$. We set $X=\mathbf{H}_{0}^{1}(\Omega) \times L_{0}^{2}(\Omega) \times \mathbf{H}_{0}^{1}(\Omega) \times L_{0}^{2}(\Omega)$ and $Y=\mathbf{H}^{-1}(\Omega) \times$ $\mathbf{L}^{2}(\Omega) \times \mathbf{H}^{-1}(\Omega)$. We define the linear operator $T \in L(Y ; X)$ as follows: 
$T(\eta, \xi, \tau)=\left(\mathbf{u}_{h}, p_{h}, \mathbf{v}_{h}, q_{h}\right)$ for $(\eta, \xi, \tau) \in Y$ and $\left(\mathbf{u}_{h}, p_{h}, \mathbf{v}_{h}, q_{h}\right) \in X$, if and only if

$$
\begin{aligned}
a\left(\mathbf{u}_{h}, \mathbf{w}\right)+b\left(\mathbf{w}, p_{h}\right) & =\langle\eta, \mathbf{w}\rangle+(\xi, \mathbf{w}) \quad \forall \mathbf{w} \in \mathbf{H}_{0}^{1}(\Omega), \\
b\left(\mathbf{u}_{h}, r\right) & =0 \quad \forall r \in L_{0}^{2}(\Omega) \\
a\left(\mathbf{v}_{h}, \mathbf{z}\right)+b\left(\mathbf{z}, q_{h}\right) & =\langle\tau, \mathbf{z}\rangle \quad \forall \mathbf{z} \in \mathbf{H}_{0}^{1}(\Omega) \\
b\left(\mathbf{v}_{h}, s\right) & =0 \quad \forall s \in L_{0}^{2}(\Omega) .
\end{aligned}
$$

We set $X_{h}=\mathbf{V}_{h}^{0} \times Q_{h}^{0} \times \mathbf{V}_{h}^{0} \times Q_{h}^{0}$. We define the discrete operator $T_{h} \in$ $L\left(Y ; X_{h}\right)$ as follows:

$$
T_{h}(\eta, \xi, \tau)=\left(\hat{\mathbf{u}}_{h}, \hat{p}_{h}, \hat{\mathbf{v}}_{h}, \hat{q}_{h}\right) \text { for }(\eta, \xi, \tau) \in Y \text { and }\left(\hat{\mathbf{u}}_{h}, \hat{p}_{h}, \hat{\mathbf{v}}_{h}, \hat{q}_{h}\right) \in X_{h}
$$

if and only if

$$
\begin{aligned}
a\left(\hat{\mathbf{u}}_{h}, \mathbf{w}_{h}\right)+b\left(\mathbf{w}_{h}, \hat{p}_{h}\right) & =\left\langle\eta, \mathbf{w}_{h}\right\rangle+\left(\xi, \mathbf{w}_{h}\right) \quad \forall \mathbf{w}_{h} \in \mathbf{V}_{h}^{0}, \\
b\left(\hat{\mathbf{u}}_{h}, r_{h}\right) & =0 \quad \forall r_{h} \in Q_{h}^{0}, \\
a\left(\mathbf{z}_{h}, \hat{\mathbf{v}}_{h}\right)+b\left(\mathbf{z}_{h}, \hat{q}_{h}\right) & =\left\langle\tau, \mathbf{z}_{h}\right\rangle \quad \forall \mathbf{z}_{h} \in \mathbf{V}_{h}^{0}, \\
b\left(\hat{\mathbf{v}}_{h}, s_{h}\right) & =0 \quad \forall s_{h} \in Q_{h}^{0} .
\end{aligned}
$$

Let $\Lambda$ denote a compact subset of $\mathbb{R}_{+}$. Next, we define $G: \Lambda \times X \rightarrow Y$ as follows:

$$
G\left(\lambda,\left(\mathbf{u}_{h}, p_{h}, \mathbf{v}_{h}, q_{h}\right)\right)=\lambda\left(\delta^{-1} \mathbf{v}_{h},-\sigma \dot{W}_{h}, \mathbf{U}_{d}-\mathbf{u}_{h}\right) .
$$

It is easily seen that the reduced optimality system (9) is equivalent to

$$
\left(\mathbf{u}_{h}, \lambda p_{h}, \mathbf{v}_{h}, \lambda q_{h}\right)+T G\left(\lambda,\left(\mathbf{u}_{h}, \lambda p_{h}, \mathbf{v}_{h}, \lambda q_{h}\right)\right)=0,
$$

and that the discrete optimality system (10) is equivalent to

$$
\left(\hat{\mathbf{u}}_{h}, \lambda \hat{p}_{h}, \hat{\mathbf{v}}_{h}, \lambda \hat{q}_{h}\right)+T_{h} G\left(\lambda,\left(\hat{\mathbf{u}}_{h}, \lambda \hat{p}_{h}, \hat{\mathbf{v}}_{h}, \lambda \hat{q}_{h}\right)\right)=0 .
$$

We define a space $Z=\mathbf{L}^{2}(\Omega) \times \mathbf{L}^{2}(\Omega) \times \mathbf{L}^{2}(\Omega)$. Then clearly this space is continuously embedded into $Y=\mathbf{H}^{-1}(\Omega) \times \mathbf{L}^{2}(\Omega) \times \mathbf{H}^{-1}(\Omega)$.

Denote the Fréchet derivative of $G(\lambda,(\mathbf{u}, p, \mathbf{v}, q))$ with respect to $(\mathbf{u}, p, \mathbf{v}, q)$ by $D G(\lambda,(\mathbf{u}, p, \mathbf{v}, q))$ or $G_{(\mathbf{u}, p, \mathbf{v}, q)}(\lambda,(\mathbf{u}, p, \mathbf{v}, q))$. Then for $\left(\mathbf{u}_{h}, p_{h}, \mathbf{v}_{h}, q_{h}\right) \in X$, we obtain

$$
\begin{aligned}
& D G\left(\lambda,\left(\mathbf{u}_{h}, p_{h}, \mathbf{v}_{h}, q_{h}\right)\right) \cdot\left(\tilde{\mathbf{u}}_{h}, \tilde{p}_{h}, \tilde{\mathbf{v}}_{h}, \tilde{q}_{h}\right) \\
= & \lambda\left(\delta^{-1} \tilde{\mathbf{v}}_{h}, 0,-\tilde{\mathbf{u}}_{h}\right) \forall\left(\tilde{\mathbf{u}}_{h}, \tilde{p}_{h}, \tilde{\mathbf{v}}_{h}, \tilde{q}_{h}\right) \in X .
\end{aligned}
$$

Proposition 3.1. $D G\left(\lambda,\left(\mathbf{u}_{h}, p_{h}, \mathbf{v}_{h}, q_{h}\right)\right) \in L(X ; Z)$ for all $\left(\mathbf{u}_{h}, p_{h}, \mathbf{v}_{h}, q_{h}\right) \in$ $X$.

Proof. It is clear that

$$
\begin{aligned}
\left\|D G\left(\lambda,\left(\mathbf{u}_{h}, p_{h}, \mathbf{v}_{h}, q_{h}\right)\right) \cdot\left(\tilde{\mathbf{u}}_{h}, \tilde{p}_{h}, \tilde{\mathbf{v}}_{h}, \tilde{q}_{h}\right)\right\|_{Z}^{2} & =\delta^{-1}\left\|\tilde{\mathbf{v}}_{h}\right\|^{2}+\left\|\tilde{\mathbf{u}}_{h}\right\|^{2} \\
& \leq C\left(\left\|\tilde{\mathbf{u}}_{h}\right\|_{1}^{2}+\left\|\tilde{\mathbf{v}}_{h}\right\|_{1}^{2}\right)
\end{aligned}
$$

Therefore, $D G\left(\lambda,\left(\mathbf{u}_{h}, p_{h}, \mathbf{v}_{h}, q_{h}\right)\right) \in L(X ; Z)$ for all $\left(\mathbf{u}_{h}, p_{h}, \mathbf{v}_{h}, q_{h}\right) \in X$. 
Proposition 3.2. $G$ is twice continuously differentiable and $D^{2} G$ is bounded on all bounded sets of $X$.

Proof. For any $\left(\mathbf{u}_{h}, p_{h}, \mathbf{v}_{h}, q_{h}\right) \in X$,

$$
D^{2} G\left(\lambda,\left(\mathbf{u}_{h}, p_{h}, \mathbf{v}_{h}, q_{h}\right)\right) \cdot\left(\tilde{\mathbf{u}}_{h}, \tilde{p}_{h}, \tilde{\mathbf{v}}_{h}, \tilde{q}_{h}\right)=(0,0,0) \quad \forall\left(\tilde{u}_{h}, \tilde{p}_{h}, \tilde{\mathbf{v}}_{h}, \tilde{q}_{h}\right) \in X .
$$

Thus, it is easy to show that $D^{2} G$ is well defined, continuous, and bounded on all bounded sets of $X$.

Proposition 3.3. For any $(\eta, \xi, \tau) \in Y,\left\|\left(T-T_{h}\right)(\eta, \xi, \tau)\right\|_{X} \rightarrow 0$ as $h \rightarrow 0$.

Proof.

$$
\begin{aligned}
\left\|\left(T-T_{h}\right)(\eta, \xi, \tau)\right\|_{X}^{2} & =\left\|\left(\mathbf{u}_{h}-\hat{\mathbf{u}}_{h}, p_{h}-\hat{p}_{h}, \mathbf{v}_{h}-\hat{\mathbf{v}}_{h}, q_{h}-\hat{q}_{h}\right)\right\|_{X}^{2} \\
& =\left\|\mathbf{u}_{h}-\hat{\mathbf{u}}_{h}\right\|_{1}^{2}+\left\|p_{h}-\hat{p}_{h}\right\|^{2}+\left\|\mathbf{v}_{h}-\hat{\mathbf{v}}_{h}\right\|_{1}^{2}+\left\|q_{h}-\hat{q}_{h}\right\|^{2} \\
& \leq C h^{2}\left(\|\eta\|^{2}+\|\xi\|^{2}+\|\tau\|^{2}\right) .
\end{aligned}
$$

Hence we have $\left\|\left(T-T_{h}\right)(\eta, \xi, \tau)\right\|_{X} \rightarrow 0$ as $h \rightarrow 0$.

Proposition 3.4. $\left\|T-T_{h}\right\|_{L(Z, X) \rightarrow 0}$ as $h \rightarrow 0$.

Proof. Note that for $(\eta, \xi, \tau) \in Z$, we have

$$
\begin{aligned}
\left\|\left(T-T_{h}\right)(\eta, \xi, \tau)\right\|_{X}^{2} & =\left\|\left(\mathbf{u}_{h}-\hat{\mathbf{u}}_{h}, p_{h}-\hat{p}_{h}, \mathbf{v}_{h}-\hat{\mathbf{v}}_{h}, q_{h}-\hat{q}_{h}\right)\right\|_{X}^{2} \\
& =\left\|\mathbf{u}_{h}-\hat{\mathbf{u}}_{h}\right\|_{1}^{2}+\left\|p_{h}-\hat{p}_{h}\right\|^{2}+\left\|\mathbf{v}_{h}-\hat{\mathbf{v}}_{h}\right\|_{1}^{2}+\left\|q_{h}-\hat{q}_{h}\right\|^{2} \\
& \leq C h^{2}\left(\|\eta\|^{2}+\|\xi\|^{2}+\|\tau\|^{2}\right) \\
& \leq C h^{2}\|(\eta, \xi, \tau)\|_{Z}^{2} .
\end{aligned}
$$

Thus, we obtain

$$
\left\|\left(T-T_{h}\right)(\eta, \xi, \tau)\right\|_{L(Z, X)}^{2}=\sup _{\|(\eta, \xi, \tau)\|_{Z}^{2} \neq 0} \frac{\left\|\left(T-T_{h}\right)(\eta, \xi, \tau)\right\|_{X}^{2}}{\|(\eta, \xi, \tau)\|_{Z}^{2}} \leq C h^{2} \rightarrow 0
$$

as $h \rightarrow 0$.

Proposition 3.5. $\left\{\left(\lambda, \psi_{h}(\lambda)\right): \lambda \in \Lambda\right\}$ is a branch of nonsingular (regular) solutions of (11).

Proof.

$$
\begin{aligned}
a\left(\check{\mathbf{u}}_{h}, \tilde{\mathbf{v}}_{h}\right)+\lambda b\left(\tilde{\mathbf{v}}_{h}, \check{p}_{h}\right)-\lambda\left(-\frac{\check{\mathbf{v}}_{h}}{\delta}, \tilde{\mathbf{v}}_{h}\right) & =\left(\mathbf{w}, \tilde{\mathbf{v}}_{h}\right) \quad \forall \tilde{\mathbf{v}}_{h} \in \mathbf{H}_{0}^{1}(\Omega), \\
b\left(\check{\mathbf{u}}_{h}, \tilde{q}_{h}\right) & =\left(l, \tilde{q}_{h}\right) \quad \forall \tilde{q}_{h} \in L_{0}^{2}(\Omega), \\
a\left(\tilde{\mathbf{u}}_{h}, \check{\mathbf{v}}_{h}\right)+\lambda b\left(\tilde{\mathbf{u}}_{h}, \check{q}_{h}\right)-\lambda\left(\check{\mathbf{u}}_{h}-\mathbf{U}_{d}, \tilde{\mathbf{u}}_{h}\right) & =\left(\mathbf{z}, \tilde{\mathbf{u}}_{h}\right) \quad \forall \tilde{\mathbf{u}}_{h} \in \mathbf{H}_{0}^{1}(\Omega), \\
b\left(\check{\mathbf{v}}_{h}, \tilde{p}_{h}\right) & =\left(m, \tilde{p}_{h}\right) \quad \forall \tilde{p}_{h} \in L_{0}^{2}(\Omega)
\end{aligned}
$$

has a unique solution $\left(\check{\mathbf{u}}_{h}, \check{p}_{h}, \check{\mathbf{v}}_{h}, \check{q}_{h}\right) \in X$ for every $\mathbf{w}, \mathbf{z} \in \mathbf{H}^{-1}(\Omega), l, m$ $\in L^{2}(\Omega)$. 
Through Propositions 3.1-3.5 we have verified all of the assumptions of Theorem 3.6. Thus, we obtain the following results.

Theorem 3.6. Let $F\left(\lambda, \psi_{h}\right)=\mathbf{0}$ denote abstract form (11) and assume that $\left\{\left(\lambda, \psi_{h}(\lambda)\right) \mid \lambda \in \Lambda\right\}$ is a branch of regular solutions of (11). Furthermore, assume that $T \in L(Y, X)$, that $G$ is a $C^{2}$ map $\Lambda \times X \mapsto Y$ such that all second derivatives of $G$ are bounded on bounded subsets of $\Lambda \times X$, and that there exists a space $Z \subset Y$, with continuous imbedding, such that $D_{\psi_{h}} G\left(\lambda, \psi_{h}\right) \in L(X, Z)$ for all $\lambda \in \Lambda$ and $\psi_{h} \in X$. If approximate problem (12) is such that

$$
\lim _{h \rightarrow 0}\left\|\left(T-T_{h}\right) \mathbf{g}\right\|_{X}=0
$$

for all $\mathbf{g} \in Y$ and

$$
\lim _{h \rightarrow 0}\left\|\left(T-T_{h}\right)\right\|_{L(Z, X)}=0 .
$$

Then

1. there exists a neighborhood $\mathcal{O}$ of the origin in $X$ and, for $h$ sufficiently small, a unique $C^{2}$ function $\lambda \mapsto \hat{\psi}_{h}(\lambda) \in X_{h}$ such that $\left\{\left(\lambda, \hat{\psi}_{h}(\lambda)\right) \mid \lambda \in \Lambda\right\}$ is a branch of regular solutions of discrete problem (12) and $\psi_{h}(\lambda)-\hat{\psi}_{h}(\lambda) \in \mathcal{O}$ for all $\lambda \in \Lambda$;

2. for all $\lambda \in \Lambda$ we have

$$
\left\|\hat{\psi}_{h}(\lambda)-\psi_{h}(\lambda)\right\|_{X} \leq C\left\|\left(T-T_{h}\right) G\left(\lambda, \psi_{h}(\lambda)\right)\right\|_{X} .
$$

Therefore, from (13), we obtain the following error estimates.

$$
\begin{aligned}
& \left\|\mathbf{u}_{h}-\hat{\mathbf{u}}_{h}\right\|_{1}+\left\|p_{h}-\hat{p}_{h}\right\|+\left\|\mathbf{v}_{h}-\hat{\mathbf{v}}_{h}\right\|_{1}+\left\|q_{h}-\hat{q}_{h}\right\| \\
\leq & C h\left(\left\|\mathbf{u}_{h}\right\|_{2}+\left\|p_{h}\right\|_{1}+\left\|\mathbf{v}_{h}\right\|_{2}+\left\|q_{h}\right\|_{1}\right) .
\end{aligned}
$$

Remark 3.7. It is supposed that the solution $(\mathbf{u}, p)$ for the optimal control problem with the functional (1) and the equations (2) and the finite element solution $\left(\hat{\mathbf{u}}_{h}, \hat{p}_{h}\right)$ for the optimality system (10) satisfy the following error estimates

$$
\begin{aligned}
& \mathbb{E}\left(\left\|\mathbf{u}-\hat{\mathbf{u}}_{h}\right\|+\left\|p-\hat{p}_{h}\right\|_{-1}\right) \\
\leq & \mathbb{E}\left(\left\|\mathbf{u}-\mathbf{u}_{h}\right\|+\left\|p-p_{h}\right\|_{-1}\right)+\mathbb{E}\left(\left\|\mathbf{u}_{h}-\hat{\mathbf{u}}_{h}\right\|+\left\|p_{h}-\hat{p}_{h}\right\|_{-1}\right) \\
\leq & C|\ln h| h^{2}+C h\left(\left\|\mathbf{u}_{h}\right\|_{1}+\left\|p_{h}\right\|\right) .
\end{aligned}
$$

\section{Numerical experiments}

In this section we will present numerical experiments using the finite element method described in Sections 2 and 3. We construct the finite dimensional subspaces $\mathbf{V}_{h}$ and $Q_{h}$ using the Taylor-Hood method. We will compute both cases of the control problems of deterministic and stochastic Stokes equations and compare the results.

We consider the optimality system with $\Omega=[0,1] \times[0,1]$, the viscous constant $\nu=1$ and the desired velocity $\mathbf{U}_{d}(x, y)=\left(u_{1}(x, y), u_{2}(x, y)\right)$ is chosen 
TABLE 1 . The norms $\left\|\mathbf{u}-\mathbf{U}_{d}\right\|,\|\mathbf{f}\|$ and $\mathcal{J}_{D}(\mathbf{u}, p, \mathbf{f})$ when $h=1 / 16$

\begin{tabular}{|c||c|c|c|}
\hline$\delta$ & $\left\|\mathbf{u}-\mathbf{U}_{d}\right\|$ & $\|\mathbf{f}\|$ & $\mathcal{J}_{D}(\mathbf{u}, p, \mathbf{f})$ \\
\hline \hline 1 & $1.2742 \mathrm{E}-01$ & $2.3711 \mathrm{E}-03$ & $8.1204 \mathrm{E}-03$ \\
$10^{-3}$ & $9.5121 \mathrm{E}-02$ & $1.7479 \mathrm{E}+00$ & $6.0515 \mathrm{E}-03$ \\
$10^{-6}$ & $9.0166 \mathrm{E}-04$ & $7.3580 \mathrm{E}+00$ & $2.7477 \mathrm{E}-05$ \\
$10^{-9}$ & $5.0727 \mathrm{E}-06$ & $7.5122 \mathrm{E}+00$ & $2.8229 \mathrm{E}-08$ \\
$10^{-12}$ & $4.5747 \mathrm{E}-06$ & $7.5128 \mathrm{E}+00$ & $3.8685 \mathrm{E}-11$ \\
$10^{-15}$ & $4.5747 \mathrm{E}-06$ & $7.5259 \mathrm{E}+00$ & $1.0492 \mathrm{E}-11$ \\
\hline
\end{tabular}

TABLE 2. The norms $\left\|\mathbf{u}-\mathbf{U}_{d}\right\|,\|\mathbf{f}\|$ and $\mathcal{J}_{D}(\mathbf{u}, p, \mathbf{f})$ when $h=1 / 32$

\begin{tabular}{|c||c|c|c|}
\hline$\delta$ & $\left\|\mathbf{u}-\mathbf{U}_{d}\right\|$ & $\|\mathbf{f}\|$ & $\mathcal{J}_{D}(\mathbf{u}, p, \mathbf{f})$ \\
\hline \hline 1 & $1.2741 \mathrm{E}-01$ & $2.3710 \mathrm{E}-03$ & $8.1200 \mathrm{E}-03$ \\
$10^{-3}$ & $9.5118 \mathrm{E}-02$ & $1.7478 \mathrm{E}+00$ & $6.0512 \mathrm{E}-03$ \\
$10^{-6}$ & $9.0305 \mathrm{E}-04$ & $7.3565 \mathrm{E}+00$ & $2.7467 \mathrm{E}-05$ \\
$10^{-9}$ & $5.5020 \mathrm{E}-06$ & $7.5457 \mathrm{E}+00$ & $2.8484 \mathrm{E}-08$ \\
$10^{-12}$ & $2.7540 \mathrm{E}-07$ & $7.5499 \mathrm{E}+00$ & $2.8538 \mathrm{E}-11$ \\
$10^{-15}$ & $2.7533 \mathrm{E}-07$ & $7.6442 \mathrm{E}+00$ & $6.7120 \mathrm{E}-14$ \\
\hline
\end{tabular}

TABLE 3. The norms $\left\|\mathbf{u}-\mathbf{U}_{d}\right\|,\|\mathbf{f}\|$ and $\mathcal{J}_{D}(\mathbf{u}, p, \mathbf{f})$ with convergence rate for $\delta=10^{-15}$

\begin{tabular}{|c||cc|c|cc|}
\hline$h$ & \multicolumn{2}{|c|}{$\left\|\mathbf{u}-\mathbf{U}_{d}\right\|$} & $\|\mathbf{f}\|$ & \multicolumn{2}{|c|}{$\mathcal{J}_{D}(\mathbf{u}, p, \mathbf{f})$} \\
\hline \hline $1 / 4$ & $1.1557 \mathrm{E}-03$ & & $7.2595 \mathrm{E}+00$ & $6.6779 \mathrm{E}-07$ & \\
$1 / 8$ & $7.6032 \mathrm{E}-05$ & 3.93 & $7.4413 \mathrm{E}+00$ & $2.8904 \mathrm{E}-09$ & 7.85 \\
$1 / 16$ & $4.5747 \mathrm{E}-06$ & 4.05 & $7.5259 \mathrm{E}+00$ & $1.0492 \mathrm{E}-11$ & 8.11 \\
$1 / 32$ & $2.7533 \mathrm{E}-07$ & 4.05 & $7.6442 \mathrm{E}+00$ & $6.7120 \mathrm{E}-14$ & 7.29 \\
\hline
\end{tabular}

TABLE 4. The norms $\left\|\mathbb{E} \hat{\mathbf{u}}_{h}-\mathbf{U}_{d}\right\|,\left\|\mathbb{E} \hat{\mathbf{f}}_{h}\right\|$ and $\mathcal{J}\left(\hat{\mathbf{u}}_{h}, \hat{p}_{h}, \hat{\mathbf{f}}_{h}\right)$ when $h=1 / 16, M=4096$

\begin{tabular}{|c||c|c|c|}
\hline$\delta$ & $\left\|\mathbb{E} \hat{\mathbf{u}}_{h}-\mathbf{U}_{d}\right\|$ & $\left\|\mathbb{E} \hat{\mathbf{f}}_{h}\right\|$ & $\mathcal{J}\left(\hat{\mathbf{u}}_{h}, \hat{p}_{h}, \hat{f}_{h}\right)$ \\
\hline \hline 1 & $1.2801 \mathrm{E}-01$ & $2.3835 \mathrm{E}-03$ & $8.1956 \mathrm{E}-03$ \\
$10^{-3}$ & $9.4946 \mathrm{E}-02$ & $1.7442 \mathrm{E}+00$ & $6.0286 \mathrm{E}-03$ \\
$10^{-6}$ & $9.0874 \mathrm{E}-04$ & $7.3461 \mathrm{E}+00$ & $2.7395 \mathrm{E}-05$ \\
$10^{-9}$ & $5.2029 \mathrm{E}-06$ & $7.5423 \mathrm{E}+00$ & $2.8456 \mathrm{E}-08$ \\
$10^{-12}$ & $4.5752 \mathrm{E}-06$ & $7.5433 \mathrm{E}+00$ & $3.8917 \mathrm{E}-11$ \\
$10^{-15}$ & $4.5747 \mathrm{E}-06$ & $7.5387 \mathrm{E}+00$ & $1.0492 \mathrm{E}-11$ \\
\hline
\end{tabular}

where

$$
u_{1}=\frac{d}{d y} \phi(x) \phi(y) \quad \text { and } \quad u_{2}=-\frac{d}{d x} \phi(x) \phi(y)
$$


TABLE 5. The norms $\left\|\mathbb{E} \hat{\mathbf{u}}_{h}-\mathbf{U}_{d}\right\|,\left\|\mathbb{E} \hat{\mathbf{f}}_{h}\right\|$ and $\mathcal{J}\left(\hat{\mathbf{u}}_{h}, \hat{p}_{h}, \hat{\mathbf{f}}_{h}\right)$ with convergence rate for $\delta=10^{-15}$

\begin{tabular}{|c|c||cc|c|cc|}
\hline$h$ & $\mathrm{M}$ & $\left\|\mathbb{E} \hat{\mathbf{u}}_{h}-\mathbf{U}_{d}\right\|$ & $\left\|\mathbb{E} \hat{\mathbf{f}}_{h}\right\|$ & \multicolumn{2}{|c|}{$\mathcal{J}\left(\hat{\mathbf{u}}_{h}, \hat{p}_{h}, \hat{\mathbf{f}}_{h}\right)$} \\
\hline \hline $1 / 4$ & 16 & $1.1567 \mathrm{E}-03$ & & $7.2294 \mathrm{E}+00$ & $6.6779 \mathrm{E}-07$ & \\
$1 / 8$ & 256 & $7.6032 \mathrm{E}-05$ & 3.93 & $7.4728 \mathrm{E}+00$ & $2.8904 \mathrm{E}-09$ & 7.85 \\
$1 / 16$ & 4096 & $4.5747 \mathrm{E}-06$ & 4.05 & $7.5016 \mathrm{E}+00$ & $1.0492 \mathrm{E}-11$ & 8.11 \\
$1 / 32$ & 65536 & $2.7638 \mathrm{E}-07$ & 4.05 & $7.5800 \mathrm{E}+00$ & $6.6921 \mathrm{E}-14$ & 7.29 \\
\hline
\end{tabular}

and

$$
\phi(z)=(1-z)^{2}(1-\cos (\pi z)) .
$$

First, we consider the control problem of deterministic Stokes equations. Since $\mathbb{E} \dot{W}=0$ and the problem is linear, $(\mathbb{E} \mathbf{u}, \mathbb{E} p, \mathbb{E} \mathbf{f})$ is the optimal solution of the deterministic control problem of the Stokes equations without white noise which is the optimal solution of the expectation of problem (1) and (2). Thus, we want to seek $(\mathbf{u}, p, \mathbf{f})$ which satisfies the minimization problem: minimize the functional

$$
\mathcal{J}_{D}(\mathbf{u}, p, \mathbf{f})=\frac{1}{2} \int\left|\mathbf{u}-\mathbf{U}_{d}\right|^{2} d x+\frac{\delta}{2} \int|\mathbf{f}|^{2} d x
$$

subject to the steady-state Stokes equations:

$$
\begin{aligned}
-\Delta \mathbf{u}+\nabla p=\mathbf{f} & \text { in } \Omega, \\
\nabla \cdot \mathbf{u}=0 & \text { in } \Omega, \\
\mathbf{u}=\mathbf{0} & \text { on } \partial \Omega .
\end{aligned}
$$

As shown in Table 1 and Table 2, one can see that the $L^{2}$ error $\left\|\mathbf{u}-\mathbf{U}_{d}\right\|$ and cost $\mathcal{J}_{D}(\mathbf{u}, p, \mathbf{f})$ go to zero as $\delta$ goes to zero in each case $h=1 / 16$ and $h=1 / 32$.

For the case $\delta=10^{-15}$, Table 3 shows the numerical results with the convergence rates.

Before solving the optimal control problem for the stochastic case, we present some random velocities with white noise and the target velocity $\mathbf{U}_{d}$ in Figure 1. We want to control these random velocities to be the target velocity $\mathbf{U}_{d}$. We set $\sigma=1$. The numerical algorithm consists of three steps.

Step 1 For $m=1, \ldots, M$, generate samples $\dot{W}_{h}^{m}=\sum_{T \in T_{h}}|T|^{-\frac{1}{2}} \xi_{T}^{m} \chi_{T}(x)$, of discretized white noise $\dot{W}_{h}$ by generating samples $\left\{\xi_{T}^{m}\right\}_{T \in T_{h}}$ of $\left\{\xi_{T}\right\}_{T \in T_{h}}$ where $M$ is the sample size;

Step 2 For $m=1, \ldots, M$, solve (10), with $\dot{W}_{h}$ replaced by $\dot{W}_{h}^{m}$, to obtain the approximate solutions $\left(\hat{\mathbf{u}}_{h}^{m}, \hat{p}_{h}^{m}, \hat{\mathbf{v}}_{h}^{m}, \hat{q}_{h}^{m}, \hat{\mathbf{f}}_{h}^{m}\right)$ of $\left(\hat{\mathbf{u}}_{h}, \hat{p}_{h}, \hat{\mathbf{v}}_{h}, \hat{q}_{h}, \hat{\mathbf{f}}_{h}\right)$ by the finite element method; 

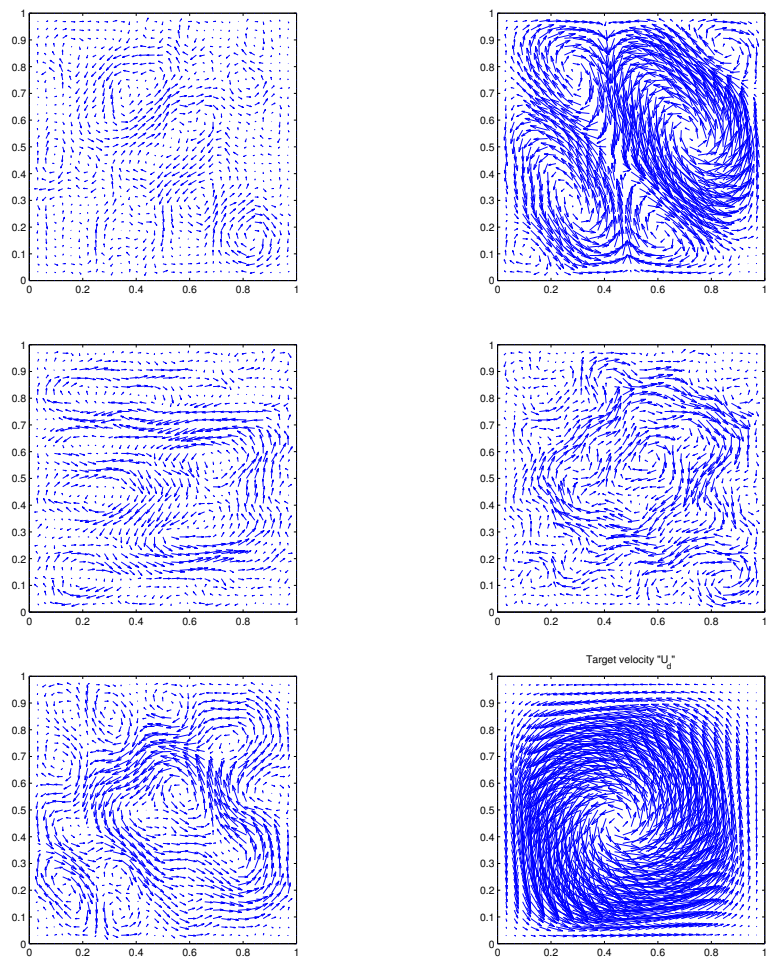

FiguRE 1. Different kinds of random velocities of stochastic Stokes equations with white noise and the target velocity $\mathbf{U}_{d}$ (bottom right)

Step 3 Evaluate statistics $\mathbb{E}\left(\mathbf{v}\left(\hat{\mathbf{u}}_{h}, \hat{p}_{h}, \hat{\mathbf{v}}_{h}, \hat{q}_{h}, \hat{\mathbf{f}}_{h}\right)\right)$ using the Monte Carlo method:

$$
\mathbb{E}\left(\mathbf{v}\left(\hat{\mathbf{u}}_{h}, \hat{p}_{h}, \hat{\mathbf{v}}_{h}, \hat{q}_{h}, \hat{\mathbf{f}}_{h}\right)\right) \approx \frac{1}{M} \sum_{m=1}^{M} \mathbf{v}\left(\hat{\mathbf{u}}_{h}^{m}, \hat{p}_{h}^{m}, \hat{\mathbf{v}}_{h}^{m}, \hat{q}_{h}^{m}, \hat{\mathbf{f}}_{h}^{m}\right) .
$$

We first discover, as shown in Figure 2, that the variance/standard deviation of the velocity $\mathbf{u}=\left(u_{1}, u_{2}\right)$ is quite small (in the order of $10^{-3}$ ), which indicates that we can perform Monte Carlo simulations with relatively small sample sizes.

In Table 4, we can see that the $L^{2}$ error $\left\|\mathbb{E} \hat{\mathbf{u}}_{h}-\mathbf{U}_{d}\right\|$ and cost $\mathcal{J}\left(\hat{\mathbf{u}}_{h}, \hat{p}_{h}, \hat{\mathbf{f}}_{h}\right)$ also go to zero as $\delta$ goes to zero in case the grid size $h=1 / 16$ and the sample size $M=4096$. In comparison with Table 1 , the values converge alike.

In Table 5 , we list the $L^{2}$ errors and convergence rates of $\left\|\mathbb{E} \hat{\mathbf{u}}_{h}-\mathbf{U}_{d}\right\|$ and $\operatorname{cost} \mathcal{J}\left(\hat{\mathbf{u}}_{h}, \hat{p}_{h}, \hat{\mathbf{f}}_{h}\right)$ when $h=1 / 16, M=4096$. We get the same convergence 

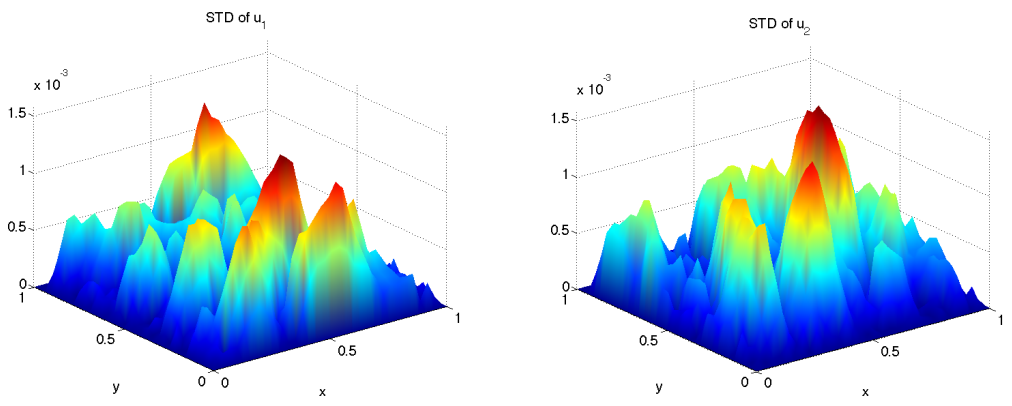

FiguRE 2. Approximate standard deviation of uncontrolled velocity $u=\left(u_{1}, u_{2}\right)$ when $h=1 / 16$, sample size $M=4096$
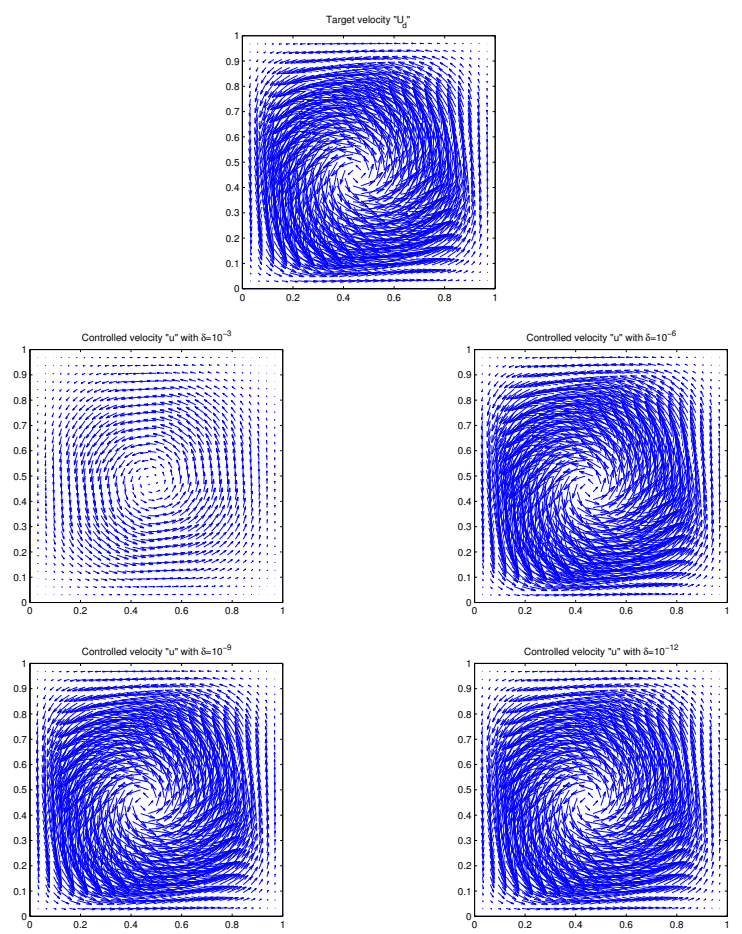

Figure 3 . Target veolicty $U_{d}$ (top), controlled velocities $\mathbb{E} \mathbf{u}_{h}$ for different values of $\delta ; \delta=10^{-3}, 10^{-6}, 10^{-9}, 10^{-12}$ (from top to bottom and left to right) when $M=4096$ and $h=1 / 16$

rates as Table 3 . We use the Monte Carlo simulations here in order to verify that sufficient number of samples have been used. 
In Figure 3, we display the target velocity and approximate velocities for $\delta=10^{-3}, 10^{-6}, 10^{-9}, 10^{-12}$ when $h=1 / 16$ is fixed.

\section{References}

[1] F. Abergal and R. Temmam, On some control problems in fluid mechanics, Theoret. Comput. Fluid Dynamics 1 (1990), 303-325.

[2] R. Adams, Sobolev Spaces, Academic Press, New York, 1975.

[3] I. Babuska and P. Chatzipantelidis, On solving elliptic stochastic partial differential equations, Comput. Methods Appl. Mech. Engrg. 191 (2002), no. 37-38, 4093-4122.

[4] I. Babuska, R. Tempone, and G. E. Zouraris, Galerkin finite element approximations of stochastic elliptic partial differential equations, SIAM J. Numer. Anal. 42 (2004), no. 2, 800-825.

[5] _ Solving elliptic boundary value problems with uncertain coefficients by the finite element method: The stochastic formulation, Comput. Methods Appl. Mech. Engrg. 194 (2005), no. 12-16, 1251-1294.

[6] F. Brezzi, J. Rappaz, and P. Raviart, Finite-dimensional approximation of nonlinear problems. I. Branches of nonsingular solutions, Numer. Math. 36 (1980), 1-25.

[7] Y. Cao, Z. Chen, and M. Gunzburger, Error analysis of finite element approximations of the stochastic Stokes equations, Adv. Comput. Math. 33 (2010), no. 2, 215-230.

[8] Y. Cao, H. Yang, and L. Yin, Finite element methods for semilinear elliptic stochastic partial differential equations, Numer. Math. 106 (2007), no. 2, 181-198.

[9] P. Ciarlet, Finite Element Method for Elliptic Problems, North-Holland, Amsterdam, 1978.

[10] _ Introduction to Numerical Linear Algebra and Optimization, Cambridge, 1989.

[11] Y. Choi, H.-C. Lee, and S. D. Kim, Analysis and computations of least-squares method for optimal control problems for the Stokes equations, J. Korean Math. Soc. 46 (2009), no. 5, 1007-1025.

[12] Y. Choi, H.-C. Lee, and B.-C. Shin, A least-square/penalty method for distributed optimal control problems for Stokes equations, Comput. Math. Appl. 53 (2007), no. 11, 1672-1685.

[13] R. G. Ghanem and P. D. Spanos, Stochasic Finite Elements: A spectral approach, Springer-Verlag, 1991.

[14] V. Girault and P.-A. Raviart, Finite Element Methods for Navier-Stokes Equations, Springer, Berlin, 1986.

[15] M. D. Gunzburger and L. S. Hou, Finite-dimensional approximation of a class of constrained nonlinear optimal control problems, SIAM J. Control. Optim. 34 (1996), no. 3, 1001-1043.

[16] M. D. Gunzburger, L. S. Hou, and T. P. Svobodny, Analysis and finite element approximation of optimal control problems for the stationary Navier-Stokes equations with distributed and Neumann controls, Math. Comp. 57 (1991), no. 195, 123-151.

[17] M. Gunzburger, H.-C. Lee, and J. Lee, Error estimates of stochastic optimal neumann boundary control problems, SIAM J. Numer. Anal. 49 (2011), no. 4, 1532-1552.

[18] M. D. Gunzburger and S. Manservisi, Analysis and approximation of the velocity tracking problem for Navier-Stokes flows with distributed control, SIAM J. Numer. Anal. 37 (2000), no. 5, 1481-1512.

[19] L. S. Hou, J. Lee, and H. Manouzi, Finite element approximations of stochastic optimal control problems constrained by stochastic elliptic PDEs, J. Math. Anal. Appl. 384 (2011), no. 1, 87-103.

[20] H. -C. Lee, Analysis and computational methods of Dirichlet boundary optimal control problems for 2D Boussinesq equations, Adv. Comput. Math. 19 (2003), no. 1-3, 255-275. 
[21] H.-C. Lee and Y. Choi, A least-squares method for optimal control problems for a secondorder elliptic systems in two dimensions, J. Math. Anal. Appl. 242 (2000), no. 1, 105128.

[22] H.-C. Lee and O. Y. Imanuvilov, Analysis pf optimal control problems for 2D stationary Boussinesq equations, J. Math. Anal. Appl. 242 (2000), 191-211.

[23] H.-C. Lee and S. Kim, Finite element approximation and computations of optimal dirichlet boundary control problems for the boussinesq equations, J. Korean Math. Soc. 41 (2004), no. 4, 681-715.

[24] J. L. Lions, Optimal Control of Systems Governed by Partial Differential Equations, Springer, New York, 1971

[25] H. Manouzi, A finite element approximation of linear stochastic PDEs driven by multiplicative white noise, Int. J. Comput. Math. 85 (2008), no. 3-4, 527-546.

[26] H. Manouzi and L. S. Hou, An optimal control problem for stochastic linear PDEs driven by a Gaussian white noise, 629-636, Numerical Mathematics and Advanced Applications, Springer Berlin Heidelberg, 2008.

[27] G. Stefanou, The stochastic finite element method: Past, present, and future, Comput. Methods Appl. Mech. Engrg. 198 (2009), Issues 9-12, 1031-1051.

[28] R. Témam, Nonlinear Functional Analysis and Navier-Stokes Equations, SIAM, Philadelphia, 1983.

Youngmi Choi

College of Liberal Arts

ANYANG UNIVERSITY

ANYANG 430-714, KoreA

E-mail address: ymchoi93@anyang.ac.kr

SOOHYUN KIM

Department of Mathematics

AJou University

SuwON 443-749, KoreA

E-mail address: lilyssam@gmail.com

Hyung-Chun Lee

Department of Mathematics

AJOu UNIVERSITY

Suwon 443-749, KoreA

E-mail address: hclee@ajou.ac.kr 